\title{
Advanced Diffusion Strategies for Junction Formation in Germanium ${ }^{+}$
}

\author{
Davide De Salvador 1,2, , Francesco Sgarbossa 1,2, Gianluigi Maggioni 1,2, Enrico Napolitani 1,2, \\ Chiara Carraro 1,2, Sara Maria Carturan 1,2, Walter Raniero 2, Stefano Bertoldo ${ }^{2}$, \\ Ruggero Milazzo 1,2, Virginia Boldrini 1,2, Gian Andrea Rizzi ${ }^{3}$, Daniel Ricardo Napoli ${ }^{2}$ and \\ Alberto Carnera ${ }^{1,2}$ \\ Dipartimento di Fisica e Astronomia, Università di Padova, Via Marzolo 8, I-35131 Padova, Italy \\ 2 National Institute for Nuclear Physics INFN, Laboratori Nazionali di Legnaro, Viale dell'Università 2, \\ I-35020 Legnaro, Italy \\ 3 Dipartimento di Chimica, Università di Padova, Via Marzolo 1, I-35131 Padova, Italy \\ * Correspondence: davide.desalvador@unipd.it \\ + Presented at the 37th International Symposium on Dynamical Properties of Solids (DyProSo 2019), Ferrara, \\ Italy, 8-12 September 2019.
}

Published: 5 September 2019

The investigation of innovative dynamical processes for the fabrication of highly doped and high quality Ge layers is currently a hot topic in many applicative fields such as nanoelectronics, photonics and radiation detectors. Challenges that require a deep physical and material science investigation are: (i) the high electrical activation in narrow region that can be obtained by out of equilibrium processes but has not to introduce lattice damage that may deteriorate the electrical properties. High concentration of the active dopant may transform germanium in a plasmonic material for sensor applications, or in an optical active material thanks to the direct gap transition that occurs at high doping and high strain. (ii) The control of the amount of doping at nanoscale (deterministic doping) is fundamental to meet the request of nanodevices production. Traditional methods as ion implantation are difficult to manage due to statistical fluctuations. In particular, this task has to be solved in germanium to exploit such material as a high mobility material in nanoelectronic. (iii) The preservation of the material purity during doping processes is a relevant problem especially when high purity germanium (HPGe) is used for gamma detector for nuclear spectroscopy and gamma imaging applications.

In this talk we will present some example of our recent research on germanium to contribute to the above challenges. Molecular doping process i.e., the production of monolayer self- assembled source of dopants on the devices surface is a promising way toward deterministic doping. We recently investigate the use of both $\mathrm{P}$ and $\mathrm{Sb}$ monolayer to this aim $[1,2]$. The effectiveness of such monolayers as diffusion sources is investigated. A very promising way to obtain very high doping is pulsed laser melting (PLM), this is a highly out equilibrium process that melts the extreme surface of the crystal and allows for dopant diffusion into the melt and its incorporation during fast regrowth. The application of this method to Ge allows for record activation of the dopants. Finally, we investigated the contamination induced by this laser process in the bulk of the material and we understood that it is a very promising method for doping of HPGe making possible fast and cheap processing for next generation gamma detectors [3,4]. 


\section{References}

1. Sgarbossa, F.; Carturan, S.M.; de Salvador, D.; Rizzi, G.A.; Napolitani, E.; Maggioni, G.; Raniero, W.; Napoli, D.R.; Granozzi, G.; Carnera, A. Monolayer doping of germanium by phosphorus-containing molecules. Nanotechnology 2018, 29, 465702.

2. Sgarbossa, F.; G; Maggioni; Rizzi, G.A.; Carturan, S.M.; Napolitani, E.; Raniero, W.; Carraro, C.; Bondino, F.; Píš, I.; de Salvador, D. Self-limiting Sb monolayer as a diffusion source for Ge doping. Appl. Surface Sci. 2019, 496, 143713.

3. Boldrini, V.; Maggioni, G.; Carturan, S.; Raniero, W.; Sgarbossa, F.; Milazzo, R.; Napoli, D.R.; Napolitani, E.; Camattari, R.; de Salvador, D. Characterization and modeling of thermally-induced doping contaminants in high-purity germanium. J. Phys. D Appl. Phys. 2018, 52, 035104.

4. Maggioni, G.; Carturan, S.M.; Raniero, W.; Riccetto, S.; Sgarbossa, F.; Boldrini, V.; Milazzo, R.; Napoli, D.R.; Scarpa, D.; Andrighetto, A.; Napolitani, E.; de Salvador, D. Pulsed laser di ff usion of thin holebarrier contacts in high purity germanium for gamma radiation detectors. Eur. Phys. J. A 2018, 54, 34.

(C) 2019 by the authors. Licensee MDPI, Basel, Switzerland. This article is an open access article distributed under the terms and conditions of the Creative Commons Attribution (CC BY) license (http://creativecommons.org/licenses/by/4.0/). 